Research Article

\title{
Enaminone-Derived Pyrazoles with Antimicrobial Activity
}

\author{
Mashooq Ahmad Bhat $\mathbb{D},{ }^{1}$ Mohamed A. Al-Omar, ${ }^{1}$ Ahmed M. Naglah $\mathbb{D}{ }^{2,3}$ \\ and Abdul Arif Khan ${ }^{4}$ \\ ${ }^{1}$ Department of Pharmaceutical Chemistry, College of Pharmacy, King Saud University, Riyadh 11451, Saudi Arabia \\ ${ }^{2}$ Department of Pharmaceutical Chemistry, Drug Exploration and Development Chair (DEDC), College of Pharmacy, \\ King Saud University, Riyadh 11451, Saudi Arabia \\ ${ }^{3}$ Peptide Chemistry Department, Chemical Industries Research Division, National Research Centre, 12622 Dokki, Cairo, Egypt \\ ${ }^{4}$ Department of Pharmaceutics, College of Pharmacy, King Saud University, Riyadh 11451, Saudi Arabia
}

Correspondence should be addressed to Mashooq Ahmad Bhat; mabhat@ksu.edu.sa

Received 15 April 2019; Revised 3 September 2019; Accepted 24 September 2019; Published 7 November 2019

Guest Editor: Maria Grazia Bonomo

Copyright ( 2019 Mashooq Ahmad Bhat et al. This is an open access article distributed under the Creative Commons Attribution License, which permits unrestricted use, distribution, and reproduction in any medium, provided the original work is properly cited.

\begin{abstract}
A series of pyrazoles derived from the substituted enaminones were synthesized and were evaluated for antimicrobial activity. All the compounds were characterized by the spectral data and elemental analysis. The synthesized compounds were initially screened for their antimicrobial activity against ATCC 6538, NCTC 10400, NCTC 10418, and ATCC 27853. During initial screening, compounds (P1, P6, and P11) presented significant antimicrobial activity through disc diffusion assay. These compounds were further evaluated for antimicrobial activity at different time points against Gram-positive and Gram-negative bacteria and presented significant activity for 6 hours. The activity was found to be greater against Gram-positive bacteria. In contrast at 24 hours, the activity was found only against Gram-positive bacteria except compound (P11), showing activity against both types of bacteria. Compound (P11) was found to have highest activity against both Gram-positive and Gram-negative bacteria.
\end{abstract}

\section{Introduction}

Enaminones are chemical compounds consisting of an amino group linked through a $\mathrm{C}=\mathrm{C}$ to a carbonyl group. They are versatile synthetic intermediates that combine the ambident nucleophilicity of enamines with the ambident electrophilicity of enones. They are typical push-pull ethylenes in which the amine group pushes and the carbonyl pulls electron density. The carbonyl group, conjugated to the enamine moiety, gives this system enough stability to be easily prepared, isolated, and stored under atmospheric conditions at room temperature $[1,2]$. Enaminones are the attractive intermediates for the synthesis of therapeutically active heterocycles such as quinolines, dibenzodiazepines, pyridinones, pyrazoles, oxazoles, and tetrahydrobenzoxazines [3-7]. They have been used as a starting material for the synthesis of antibacterial [8], anti-inflammatory [9], anticonvulsant [10], and antitumor agents [11]. Enaminones are versatile synthones because of their promptness to both electrophilic and nucleophilic attack [12]. They have been used in the synthesis of various heterocyclic compounds. Besides reducing the reaction time and increasing the yield and efficiency of the process, most of the research focused on a cleaner way to obtain enaminones.

Pyrazoles display a broad spectrum of potential pharmacological activities and are present in a number of pharmacologically active molecules such as phenazone, amidopyrene, methampyrone, azolid, tandearil, indoxacarb, and anturane. Pyrazoles are the most important scaffold having their broad spectrum of applications in the pharmaceutical field [13]. Pyrazole derivatives are reported to exhibit good anti-inflammatory [14], analgesic [15], anticonvulsant [16], and antidepressant activity [17]. Recently some arylpyrazoles were reported to have nonnucleoside HIV-1 reverse transcriptase inhibitor activity [18]. Nsubstituted pyrazoline derivatives also exhibit biological activities like antimicrobial [19], antitumor [20], antidepressant [21], and antitubercular activity [22]. 
In continuation of our work on pyrazoles [23], herein, we have synthesized a series of $N$-arylpyrazoles, which were derived from substituted enaminones and were found to possess an interesting profile as antimicrobial agents.

\section{Materials and Methods}

2.1. Experimental. Solvents were procured from Merck. Thin layer chromatography (TLC) was performed on Silica gel $60 \mathrm{~F}_{254}$ coated plates (Merck) to check the purity of compounds. Melting points were determined by using the Gallenkamp melting point apparatus. ${ }^{1} \mathrm{H}$ and ${ }^{13} \mathrm{C}$ NMR were recorded in the Bruker NMR $500 / 700 \mathrm{MHz}$ and 125/ $176 \mathrm{MHz}$ spectrophotometers. The samples were run in DMSO- $\mathrm{d}_{6}$ with tetramethylsilane (TMS) as an internal standard. The molecular masses of compounds were determined by Agilent triple quadrupole 6410 TQ GC/MS equipped with ESI (electrospray ionization) source. The CHN Elementar (Analysensysteme $\mathrm{GmbH}$, Germany) was used for elemental analysis of the compounds.

2.2. Synthesis of Substituted Enaminones (1-11). A mixture of substituted acetophenone $(0.02 \mathrm{~mol})$ and dimethyl formamide-dimethylacetal (DMF-DMA) (II) (0.023 mol) was refluxed for $12 \mathrm{~h}$ under solvent-free condition on a heating mantle, and then, the mixture was left to cool slowly at room temperature. The precipitate was obtained. Diethyl ether was added to the precipitate, and filtration was performed under vacuum. The obtained product was recrystallized from absolute ethanol [24].

2.3. Synthesis of Pyrazoles (P1-P11). A mixture of enaminone $(0.01 \mathrm{~mol})$ and hydrazine hydrate $99 \%$ (0.01 mol) was refluxed in absolute alcohol for 3 hours. The mixture was poured into cold water. The precipitate was obtained. The product was obtained by filtration under vacuum. The product was recrystallized from ethanol [25].

3-(2,4,6-Trimethoxyphenyl)-1H-pyrazole (P1): yield:

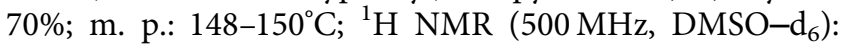
$\delta=\left(3.75\left(3 \mathrm{H}, \mathrm{s},-\mathrm{OCH}_{3}\right), 3.85\left(3 \mathrm{H}, \mathrm{s},-\mathrm{OCH}_{3}\right), 3.92(3 \mathrm{H}, \mathrm{s}\right.$, $\left.-\mathrm{OCH}_{3}\right), 6.15-6.17(2 \mathrm{H}, \mathrm{m}, \mathrm{Ar}-\mathrm{H}), 6.80(1 \mathrm{H}, \mathrm{d}, J=7 \mathrm{~Hz}$, pyrazole), $7.79(1 \mathrm{H}, \mathrm{d}, J=7 \mathrm{~Hz}$, pyrazole $), 11.81(1 \mathrm{H}, \mathrm{s}, \mathrm{NH}$, $\mathrm{D}_{2} \mathrm{O}$ exchg.); ${ }^{13} \mathrm{C}$ NMR (125.76 MHz, DMSO-d 6 ): $\delta=55.5$ (C10), 55.9 (C11, C12), 94.3 (C6), 98.9 (C8), 106.0 (C4), 106.1 (C2), 131.7 (C3), 148.0 (C1), 158.5 (C5), 158.9 (C9), 160.4 (C7); MS: $\mathrm{m} / \mathrm{z}=234.25[\mathrm{M}]^{+}$; analysis: for $\mathrm{C}_{12} \mathrm{H}_{14} \mathrm{~N}_{2} \mathrm{O}_{3}$, calcd. C 61.53, $\mathrm{H}$ 6.02, N 11.96\%; found $\mathrm{C}$ 61.77, H 6.03, N 11.99\% [26].

3-(2,4,5-Trimethoxyphenyl)-1H-pyrazole (P2): yield:

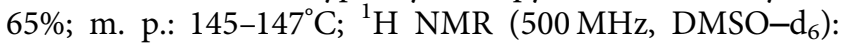
$\delta=3.78\left(3 \mathrm{H}, \mathrm{s},-\mathrm{OCH}_{3}\right), 3.85\left(3 \mathrm{H}, \mathrm{s},-\mathrm{OCH}_{3}\right), 3.86(3 \mathrm{H}, \mathrm{s}$, $\left.-\mathrm{OCH}_{3}\right), 6.65(1 \mathrm{H}, \mathrm{d}, J=7 \mathrm{~Hz}$, pyrazole $), 6.78(1 \mathrm{H}, \mathrm{d}, J=7 \mathrm{~Hz}$, pyrazole), $7.31-7.73(2 \mathrm{H}, \mathrm{m}, \mathrm{Ar}-\mathrm{H}), 12.80\left(1 \mathrm{H}, \mathrm{s}, \mathrm{NH}, \mathrm{D}_{2} \mathrm{O}\right.$ exchg.); ${ }^{13} \mathrm{C}$ NMR (125.76 MHz, DMSO-d 6 ): $\delta=56.1$ (C10, C11, C12), 98.7 (C6), 99.1 (C8), 104.2 (C4), 105.4 (C2), 129.1 (C3), 139.5 (C1), 143.2 (C5), 149.8 (C7), 150.7 (C9); MS: m/ $\mathrm{Z}=234.25[\mathrm{M}]^{+}$; analysis: for $\mathrm{C}_{12} \mathrm{H}_{14} \mathrm{~N}_{2} \mathrm{O}_{3}$ calcd. $\mathrm{C} 61.53, \mathrm{H}$ 6.02, N 11.96\%; found C 61.76, H 6.04, N 11.98\% [27].
3-(3,4-Dimethoxyphenyl)-1H-pyrazole (P3): yield: 75\%; m. p.: $123-125^{\circ} \mathrm{C} ;{ }^{1} \mathrm{H}$ NMR (500 MHz, DMSO- $\left.\mathrm{d}_{6}\right): \delta=3.78$ $\left(3 \mathrm{H}, \mathrm{s},-\mathrm{OCH}_{3}\right), 3.62\left(3 \mathrm{H}, \mathrm{s},-\mathrm{OCH}_{3}\right), 6.68(1 \mathrm{H}, \mathrm{d}, J=7 \mathrm{~Hz}$, pyrazole), $6.98(1 \mathrm{H}, \mathrm{d}, J=7 \mathrm{~Hz}$, pyrazole $), 7.33-7.70(3 \mathrm{H}, \mathrm{m}$, Ar-H), 12.78 (1H, s, NH, $\mathrm{D}_{2} \mathrm{O}$ exchg.); ${ }^{13} \mathrm{C} \quad \mathrm{NMR}$ $\left(125.76 \mathrm{MHz}, \mathrm{DMSO}-\mathrm{d}_{6}\right): \delta=55.9$ (C10, C11), 101.8 (C6), 102.0 (C8), 109.2 (C4), 118.0 (C2), 130.1 (C3), 140.4 (C1), 148.6 (C5), 149.2 (C9), 150.5 (C7); MS: m/z=204.22 [M] ${ }^{+}$; analysis: for $\mathrm{C}_{11} \mathrm{H}_{12} \mathrm{~N}_{2} \mathrm{O}_{2}$ calcd. $\mathrm{C} 64.69, \mathrm{H} 5.92, \mathrm{~N} 13.72 \%$; found $\mathrm{C} 64.84, \mathrm{H}$ 5.90, N 13.70\% [28].

4-[4-(1H-Pyrazol-3-yl)phenyl]morpholine (P4): yield: $70 \%$; m. p.: $210-212^{\circ} \mathrm{C}$; ${ }^{1} \mathrm{H}$ NMR (500 MHz, DMSO- $\left.\mathrm{d}_{6}\right)$ : $\delta=1.9\left(4 \mathrm{H}, \mathrm{s}, 2 \times \mathrm{CH}_{2}\right.$, morpholine), $3.73\left(4 \mathrm{H}, \mathrm{s}, 2 \times \mathrm{CH}_{2}\right.$, morpholine), $6.10(4 \mathrm{H}, \mathrm{m}, \mathrm{Ar}-\mathrm{H}), 6.88(1 \mathrm{H}, \mathrm{d}, J=7 \mathrm{~Hz}$, pyrazole), $7.56(1 \mathrm{H}, \mathrm{d}, J=7 \mathrm{~Hz}$, pyrazole $), 11.80(1 \mathrm{H}, \mathrm{s}, \mathrm{NH}$, $\mathrm{D}_{2} \mathrm{O}$ exchg.); ${ }^{13} \mathrm{C}$ NMR $\left(125.76 \mathrm{MHz}, \mathrm{DMSO}-\mathrm{d}_{6}\right): \delta=48.6$ (C10, C11), 66.5 (C12, C13), 114.9 (C2, C6, C8), 126.0 (C4, C9), 131.3 (C3), 143.3 (C1), 150.6 (C7); MS: m/z=229.27 $[\mathrm{M}]^{+}$; analysis: for $\mathrm{C}_{13} \mathrm{H}_{15} \mathrm{~N}_{3} \mathrm{O}$ calcd. C 68.10, $\mathrm{H} 6.59, \mathrm{~N}$ 18.33\%; found C 68.36, H 6.60, N 18.40\% [29].

1-[4-(1H-Pyrazol-3-yl)phenyl]piperidine (P5): yield: $60 \%$; m. p.: $170-172^{\circ} \mathrm{C} ;{ }^{1} \mathrm{H}$ NMR $\left(500 \mathrm{MHz}, \mathrm{DMSO}-\mathrm{d}_{6}\right)$ : $\delta=1.23$ (6H, s, piperidine), $3.16(4 \mathrm{H}$, s, piperidine), 6.0-6.98 $(4 \mathrm{H}, \mathrm{m}, \mathrm{Ar}-\mathrm{H}), 7.47(1 \mathrm{H}, \mathrm{d}, J=7 \mathrm{~Hz}$, pyrazole $), 7.63(1 \mathrm{H}, \mathrm{d}$, $J=7 \mathrm{~Hz}$, pyrazole), 12.8 (1H, s, NH, $\mathrm{D}_{2} \mathrm{O}$ exchg. $) ;{ }^{13} \mathrm{C} \mathrm{NMR}$ (125.76 MHz, DMSO- $\left.\mathrm{d}_{6}\right): \delta=24.3$ (C12), 25.6 (C13), 49.7 (C10, C11), 101.2 (C2), 116.0 (C6, C8), 126.3 (C4, C9), 151.3 (C3, C1, C7); MS: $\mathrm{m} / \mathrm{z}=227.30[\mathrm{M}]^{+}$; analysis: for $\mathrm{C}_{14} \mathrm{H}_{17} \mathrm{~N}_{3}$ calcd. C 73.98, H 7.54, N 18.49\%; found C 73.69, H 7.56, N $18.55 \%$.

3-(2,4-Dimethoxyphenyl)- $1 H$-pyrazole (P6): yield: $80 \%$; m. p.: $125-127^{\circ} \mathrm{C} ;{ }^{1} \mathrm{H}$ NMR (500 MHz, DMSO- $\left.\mathrm{d}_{6}\right): \delta=3.81$ $\left(3 \mathrm{H}, \mathrm{s},-\mathrm{OCH}_{3}\right), 3.83\left(3 \mathrm{H}, \mathrm{s},-\mathrm{OCH}_{3}\right), 6.61(1 \mathrm{H}, \mathrm{d}, J=7 \mathrm{~Hz}$, pyrazole), $6.66(1 \mathrm{H}, \mathrm{d}, J=7 \mathrm{~Hz}$, pyrazole $), 7.50-7.62(3 \mathrm{H}, \mathrm{m}$, Ar-H), 12.8 (1H, s, NH, $\mathrm{D}_{2} \mathrm{O}$ exchg.); ${ }^{13} \mathrm{C} \quad \mathrm{NMR}$ $\left(125.76 \mathrm{MHz}, \mathrm{DMSO}-\mathrm{d}_{6}\right): \delta=55.7$ (C10), 55.9 (C11), 99.1 (C6), 103.9 (C8), 105.4 (C2, C4), 105.9 (C3), 128.9 (C1), 139.4 (C5), 157.3 (9), 160.6 (C7); MS: $\mathrm{m} / \mathrm{z}=204.22[\mathrm{M}]^{+}$; analysis: for $\mathrm{C}_{11} \mathrm{H}_{12} \mathrm{~N}_{2} \mathrm{O}_{2}$ calcd. $\mathrm{C} 64.69, \mathrm{H} 5.92, \mathrm{~N} 13.72 \%$; found $\mathrm{C}$ 64.74, H 5.91, N 13.76\% [30].

3-(4-Methoxyphenyl)-1H-pyrazole (P7): yield: 90\%; m. p.: $126-128^{\circ} \mathrm{C} ;{ }^{1} \mathrm{H}$ NMR $\left(500 \mathrm{MHz}, \mathrm{DMSO}-\mathrm{d}_{6}\right): \delta=3.78(3 \mathrm{H}$, $\left.\mathrm{s},-\mathrm{OCH}_{3}\right), 6.63(1 \mathrm{H}, \mathrm{d}, J=7 \mathrm{~Hz}$, pyrazole $), 6.99(1 \mathrm{H}, \mathrm{d}$, $J=7 \mathrm{~Hz}$, pyrazole), 7.55-7.78 (4H, m, Ar-H), $12.8(1 \mathrm{H}, \mathrm{s}$, $\mathrm{NH}, \mathrm{D}_{2} \mathrm{O}$ exchg.); ${ }^{13} \mathrm{C}$ NMR $\left(125.76 \mathrm{MHz}, \mathrm{DMSO}-\mathrm{d}_{6}\right)$ : $\delta=55.5$ (C10), 101.7 (C6, C8), 114.4 (C4), 126.9 (C2), 130.1 (C3), 140.4 (C1), 142.2 (C5), 160.4 (C9), 159.1 (C7); MS: m/ $\mathrm{Z}=174.19[\mathrm{M}]^{+}$; analysis: for $\mathrm{C}_{10} \mathrm{H}_{10} \mathrm{~N}_{2} \mathrm{O}$ calcd. $\mathrm{C} 68.95, \mathrm{H}$ 5.79, N 16.08\%; found C 68.77, H 5.77, N 16.14\% [31].

3-(2,5-Dimethoxyphenyl)-1H-pyrazole (P8): yield: $80 \%$; m. p.: $108-110^{\circ} \mathrm{C} ;{ }^{1} \mathrm{H}$ NMR $\left(500 \mathrm{MHz}, \mathrm{DMSO}-\mathrm{d}_{6}\right): \delta=3.81$ $\left(3 \mathrm{H}, \mathrm{s},-\mathrm{OCH}_{3}\right), 3.83\left(3 \mathrm{H}, \mathrm{s},-\mathrm{OCH}_{3}\right), 6.61(1 \mathrm{H}, \mathrm{d}, J=7 \mathrm{~Hz}$, pyrazole), $6.66(1 \mathrm{H}, \mathrm{d}, J=7 \mathrm{~Hz}$, pyrazole $), 7.50-7.62(3 \mathrm{H}, \mathrm{m}$, Ar-H), 12.8 (1H, s, NH, $\mathrm{D}_{2} \mathrm{O}$ exchg.); ${ }^{13} \mathrm{C} \quad \mathrm{NMR}$ (125.76 MHz, DMSO- $\mathrm{d}_{6}$ ): $\delta=55.7$ (C10), 55.9 (C11), 99.1 (C6), 103.9 (C8), 105.4 (C4), 105.9 (C2), 128.9 (C3), 139.4 (C1), 157.3 (C5, C9), 160.6 (C7); MS: $\mathrm{m} / \mathrm{z}=204.22[\mathrm{M}]+$;

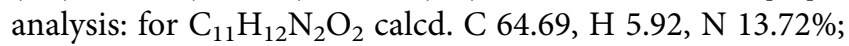
found $\mathrm{C}$ 64.87, H 5.94, N 13.77\% [32]. 
3-(2,3-Dihydro-1,4-benzodioxin-6-yl)-1 $H$-pyrazole (P9): yield: $55 \%$; m. p.: $68-70^{\circ} \mathrm{C} ;{ }^{1} \mathrm{H}$ NMR $(500 \mathrm{MHz}$, DMSO- $\left.\mathrm{d}_{6}\right): \delta=4.26\left(4 \mathrm{H}, \mathrm{s}, 2 \times \mathrm{OCH}_{2}\right), 6.62(1 \mathrm{H}, \mathrm{d}, J=7 \mathrm{~Hz}$, pyrazole), $6.88(1 \mathrm{H}, \mathrm{d}, J=7 \mathrm{~Hz}$, pyrazole $), 7.30-7.73(3 \mathrm{H}, \mathrm{m}$, Ar-H), 12.8 (1H, s, NH, $\mathrm{D}_{2} \mathrm{O}$ exchg.); ${ }^{13} \mathrm{C} \quad \mathrm{NMR}$ $\left(125.76 \mathrm{MHz}, \mathrm{DMSO}-\mathrm{d}_{6}\right): \delta=64.5$ (C10, C11), 101.8 (C6), 114.1 (C8), 117.6 (C4), 118.7 (C2), 127.8 (C3), 130.1 (C1), 143.2 (C5), 143.8 (C9), 150.1 (C7); MS: $\mathrm{m} / \mathrm{z}=202.20[\mathrm{M}]^{+}$; analysis: for $\mathrm{C}_{11} \mathrm{H}_{10} \mathrm{~N}_{2} \mathrm{O}_{2}$ calcd. C 65.34, $\mathrm{H} 4.98, \mathrm{~N} 13.85 \%$; found $\mathrm{C} 65.40, \mathrm{H} 4.96, \mathrm{~N} 13.80 \%$.

3-[4-(1H-Imidazol-1-yl)phenyl]-1H-pyrazole (P10): yield: $68 \%$; m. p.: $178-180^{\circ} \mathrm{C}$; ${ }^{1} \mathrm{H}$ NMR $(500 \mathrm{MHz}, \mathrm{DMSO}-$ $\left.\mathrm{d}_{6}\right): \delta=6.80(1 \mathrm{H}, \mathrm{d}, J=7 \mathrm{~Hz}$, pyrazole), $7.14(1 \mathrm{H}, \mathrm{d}, J=7 \mathrm{~Hz}$, pyrazole), 7.70-8.32 (7H, m, Ar-H), $12.99\left(1 \mathrm{H}, \mathrm{s}, \mathrm{NH}, \mathrm{D}_{2} \mathrm{O}\right.$ exchg.); ${ }^{13} \mathrm{C}$ NMR (125.76 MHz, DMSO- $\left.\mathrm{d}_{6}\right): \delta=102.4$ (C6), 118.3 (C8), 120.9 (C10), 126.8 (C11, C12), 130.3 (C2, C4), 130.6 (C3), 135.9 (C1), 136.3 (C5, C9), 149.6 (C7); MS: m/ $\mathrm{z}=210.23[\mathrm{M}]^{+}$; analysis: for $\mathrm{C}_{12} \mathrm{H}_{10} \mathrm{~N}_{4}$ calcd. $\mathrm{C} 68.56 \mathrm{H} 4.79$, N 26.65\%; found C 68.70, H 4.80, N 26.70\%.

3-(4-Bromophenyl)-1H-pyrazole (P11): yield: 70\%; m. p.: $148-150^{\circ} \mathrm{C} ;{ }^{1} \mathrm{H}$ NMR $\left(500 \mathrm{MHz}, \mathrm{DMSO}-\mathrm{d}_{6}\right): \delta=6.75(1 \mathrm{H}$, d, $J=7 \mathrm{~Hz}$, pyrazole $), 7.58(1 \mathrm{H}, \mathrm{d}, J=7 \mathrm{~Hz}$, pyrazole), 7.79-7.81 (4H, m, Ar-H), $13.00\left(1 \mathrm{H}, \mathrm{s}, \mathrm{NH}, \mathrm{D}_{2} \mathrm{O}\right.$ exchg.); ${ }^{13} \mathrm{C}$ NMR (125.76 MHz, DMSO-d $\left.{ }_{6}\right): \delta=102.4$ (C6), 120.8 (C8), 127.6 (C2), 130.5 (C4), 131.9 (C3), 132.8 (C1), 133.5 (C5, C9), 149.4 (C7); MS: $\mathrm{m} / \mathrm{z}=190.19[\mathrm{M}]^{+}$; analysis: for $\mathrm{C}_{10} \mathrm{H}_{10} \mathrm{~N}_{2} \mathrm{O}_{2}$ calcd. C 63.15, H 5.30, N 14.73\%; found $\mathrm{C}$ 63.35, H 5.31, N 14.78\% [33].

2.4. Synthesis of Pyrazole Derivatives (P12-P16). A mixture of pyrazole $(0.01 \mathrm{~mol})$, phenacyl bromide $(0.01 \mathrm{~mol})$, and triethylamine $(0.01 \mathrm{~mol})$ was refluxed in DMF for 10 hours. The precipitates were obtained by pouring the reaction mixture into cold water $(50 \mathrm{~mL})$. The products were obtained by filtration under vacuum. The products were washed several times with cold water. The obtained products were recrystallized from glacial acetic acid $(5 \mathrm{~mL})$ and ethanol $(100 \mathrm{~mL})$ mixture. Analytical and spectral data for the compounds were in good agreement with the expected structures of the compounds.

1-Phenyl-2-[3-(2,4,6-trimethoxyphenyl)-1H-pyrazol-1yl] ethan-1-one (P12): yield: $70 \%$; m. p.: $128-130^{\circ} \mathrm{C} ;{ }^{1} \mathrm{H}$ NMR $\left(500 \mathrm{MHz}, \mathrm{DMSO}-\mathrm{d}_{6}\right): \delta=3.69\left(6 \mathrm{H}, \mathrm{s}, 2 \times-\mathrm{OCH}_{3}\right), 3.81$ $\left(3 \mathrm{H}, \mathrm{s},-\mathrm{OCH}_{3}\right), 5.80\left(2 \mathrm{H}, \mathrm{s}, \mathrm{CH}_{2} \mathrm{O}\right), 6.19(1 \mathrm{H}, \mathrm{d}, J=7 \mathrm{~Hz}$, pyrazole), $6.27(1 \mathrm{H}, \mathrm{d}, J=7 \mathrm{~Hz}$, pyrazole), $7.58-8.0(7 \mathrm{H}, \mathrm{m}$, Ar-H); ${ }^{13} \mathrm{C}$ NMR $\left(125.76 \mathrm{MHz}, \mathrm{DMSO}-\mathrm{d}_{6}\right): \delta=55.7$ (C10), 56.1 (C11), 58.1 (C12), 91.4 (C6, C8), 105.3 (C4), 108.4 (C2), 128.5 (C16, C17), 129.3 (C20), 131.5 (C19), 134.3 (C18), 135.1 (C15), 144.3 (C1), 159.5 (C5), 161.1 (C9), 194.3 (C14); MS: $\mathrm{m} / \mathrm{z}=352.38[\mathrm{M}]^{+}$; analysis: for $\mathrm{C}_{20} \mathrm{H}_{20} \mathrm{~N}_{2} \mathrm{O}_{4}$ calcd. $\mathrm{C}$ 68.17, H 5.72, N 7.95\%; found C 68.34, H 5.70, N 7.98\%.

1-Phenyl-2-[3-(2,4,5-trimethoxyphenyl)-1H-pyrazol-1yl] ethan-1-one (P13): yield: $70 \%$; m. p.: $140-142^{\circ} \mathrm{C} ;{ }^{1} \mathrm{H}$ NMR $\left(500 \mathrm{MHz}, \mathrm{DMSO}-\mathrm{d}_{6}\right): \delta=3.70\left(3 \mathrm{H}, \mathrm{s},-\mathrm{OCH}_{3}\right), 3.83(3 \mathrm{H}, \mathrm{s}$, $\left.-\mathrm{OCH}_{3}\right), 3.86\left(3 \mathrm{H}, \mathrm{s},-\mathrm{OCH}_{3}\right) 5.92\left(2 \mathrm{H}, \mathrm{s}, \mathrm{CH}_{2} \mathrm{O}\right), 6.74(1 \mathrm{H}$, d, $J=7 \mathrm{~Hz}$, pyrazole), $7.40(1 \mathrm{H}, \mathrm{d}, J=7 \mathrm{~Hz}$, pyrazole $)$, 7.59-8.0 (6H, m, Ar-H); ${ }^{13} \mathrm{C}$ NMR $(125.76 \mathrm{MHz}$, DMSO$\left.\mathrm{d}_{6}\right): \delta=56.2$ (C10), 56.5 (C11), 58.3 (C12), 99.2 (C6), 106.6
(C8), 111.9 (C4), 113.8 (C2), 129.4 (C16), 132.8 (C17), 134.4 (C20), 134.9 (C19), 142.1 (C18), 147.6 (C15), 147.6 (C1), 149.5 (C5), 151.4 (C9), 194.3 (C14); MS: $\mathrm{m} / \mathrm{z}=352.38[\mathrm{M}]^{+}$; analysis: for $\mathrm{C}_{20} \mathrm{H}_{20} \mathrm{~N}_{2} \mathrm{O}_{4}$ calcd. C 68.17, $\mathrm{H} \mathrm{5.72,} \mathrm{N} \mathrm{7.95;}$ found $\mathrm{C} 68.30, \mathrm{H}$ 5.71, N 7.97\%.

2-[3-(3,4-Dimethoxyphenyl)-1H-pyrazol-1-yl]-1-phenylethan-1-one (P14): yield: $70 \%$; m. p.: $118-120^{\circ} \mathrm{C} ;{ }^{1} \mathrm{H}$ NMR $\left(500 \mathrm{MHz}, \mathrm{DMSO}-\mathrm{d}_{6}\right): \delta=3.78\left(3 \mathrm{H}, \mathrm{s},-\mathrm{OCH}_{3}\right), 3.80$ $\left(3 \mathrm{H}, \mathrm{s},-\mathrm{OCH}_{3}\right), 5.90\left(2 \mathrm{H}, \mathrm{s}, \mathrm{CH}_{2} \mathrm{O}\right), 6.75(1 \mathrm{H}, \mathrm{d}, J=7 \mathrm{~Hz}$, pyrazole), $6.99(1 \mathrm{H}, \mathrm{d}, J=7 \mathrm{~Hz}$, pyrazole $), 7.32-8.0(8 \mathrm{H}, \mathrm{m}$, $\mathrm{Ar}-\mathrm{H}) ;{ }^{13} \mathrm{C}$ NMR $\left(125.76 \mathrm{MHz}, \mathrm{DMSO}-\mathrm{d}_{6}\right): \delta=55.8(\mathrm{C} 10)$, 58.3 (C11), 103.0 (C6), 109.0 (C8), 112.3 (C4), 118.0 (C2), 126.7 (C16), 128.5 (C17), 129.4 (C20), 138.7 (C19), 134.4 (C18), 135.0 (C15), 148.8 (C1), 149.2 (C5), 150.8 (C9), 194.2 (C14); MS: $\mathrm{m} / \mathrm{z}=322.35[\mathrm{M}]^{+}$; analysis: for $\mathrm{C}_{19} \mathrm{H}_{18} \mathrm{~N}_{2} \mathrm{O}_{3}$ calcd. C 70.79, H 5.63, N 8.69\%; found C 70.99, H 5.65, N $8.72 \%$.

2-[3-(2,4-Dimethoxyphenyl)-1H-pyrazol-1-yl]-1-phenylethan-1-one (P15): yield: $70 \%$; m. p.: $108-110^{\circ} \mathrm{C} ;{ }^{1} \mathrm{H}$ NMR $\left(500 \mathrm{MHz}, \mathrm{DMSO}-\mathrm{d}_{6}\right): \delta=3.80\left(3 \mathrm{H}, \mathrm{s},-\mathrm{OCH}_{3}\right), 3.87$ $\left(3 \mathrm{H}, \mathrm{s},-\mathrm{OCH}_{3}\right), 5.87\left(2 \mathrm{H}, \mathrm{s}, \mathrm{CH}_{2} \mathrm{O}\right), 6.68(1 \mathrm{H}, \mathrm{d}, J=7 \mathrm{~Hz}$, pyrazole), $6.78(1 \mathrm{H}, \mathrm{d}, J=7 \mathrm{~Hz}$, pyrazole), $7.55-8.0(7 \mathrm{H}, \mathrm{m}$, $\mathrm{Ar}-\mathrm{H}) ;{ }^{13} \mathrm{C}$ NMR $\left(125.76 \mathrm{MHz}, \mathrm{DMSO}^{-} \mathrm{d}_{6}\right): \delta=55.6(\mathrm{C} 10)$, 58.2 (C11), 99.1 (C8), 105.7 (C6), 106.6 (C4), 115.2 (C2), 128.5 (C16), 128.9 (C17), 129.4 (C19), 132.5 (C20), 134.3 (C18), 135.0 (C15), 147.7 (C1), 157.8 (C5), 160.3 (C9), 194.3 (C14); MS: $\mathrm{m} / \mathrm{z}=[\mathrm{M}]+$; analysis: for $\mathrm{C}_{19} \mathrm{H}_{18} \mathrm{~N}_{2} \mathrm{O}_{3}$ calcd. $\mathrm{C}$ 70.79, H 5.63, N 8.69\%; found C 70.98, H 5.64, N 8.71\%.

2-[3-(4-Methoxyphenyl)-1 $H$-pyrazol-1-yl]-1-phenylethan-1-one (P16): yield: $70 \%$; m. p.: $75-77^{\circ} \mathrm{C} ;{ }^{1} \mathrm{H}$ NMR $\left(500 \mathrm{MHz}, \mathrm{DMSO}-\mathrm{d}_{6}\right): \delta=3.78\left(3 \mathrm{H}, \mathrm{s},-\mathrm{OCH}_{3}\right), 5.88(2 \mathrm{H}, \mathrm{s}$, $\left.\mathrm{CH}_{2} \mathrm{O}\right), 6.79(1 \mathrm{H}, \mathrm{d}, J=7 \mathrm{~Hz}$, pyrazole $), 6.98(1 \mathrm{H}, \mathrm{d}, J=7 \mathrm{~Hz}$, pyrazole), 7.59-8.0 (9H, m, Ar-H); ${ }^{13} \mathrm{C}$ NMR $(125.76 \mathrm{MHz}$, DMSO- $\left.\mathrm{d}_{6}\right): \delta=55.5$ (C10), 102.8 (C6), 114.4 (C8), 126.5 (C4, C2), 126.8 (C16), 128.5 (C17), 129.4 (C19), 133.7 (C20), 134.4 (C18), 135.0 (C15), 150.6 (C1), 159.1 (C5, C9), 194.2 (C14); MS: $\mathrm{m} / \mathrm{z}=292.33[\mathrm{M}]^{+}$; analysis: for $\mathrm{C}_{18} \mathrm{H}_{16} \mathrm{~N}_{2} \mathrm{O}_{2}$ calcd. C 73.95, H 5.52, N 9.58\%; found C 73.77, H 5.50, N 9.53\%.

2.5. Bacterial Culture. Bacterial growth inhibitory activity of synthesized compounds was checked against Gram-positive and Gram-negative bacteria. In order to test antibacterial activity of synthesized compounds, ATCC 6538, NCTC 10400 (Gram-positive); NCTC 10418, and ATCC 27853 (Gram-negative) were obtained from the microbiology unit at Department of Pharmaceutics, College of Pharmacy, King Saud University. Initially, the bacteria were grown at $37^{\circ} \mathrm{C}$ for overnight in Mueller-Hinton agar. The actively growing bacteria were transferred to Mueller-Hinton broth and further incubated overnight at $37^{\circ} \mathrm{C}$. The resultant active bacteria were used for antibacterial activity.

2.6. Screening of Synthesized Compounds for Antibacterial Activity. The synthesized compounds were initially screened for their antibacterial activity against ATCC 6538 and NCTC 10418 with the use of agar well diffusion assay [34]. The bacterial culture turbidity was established to 0.5 


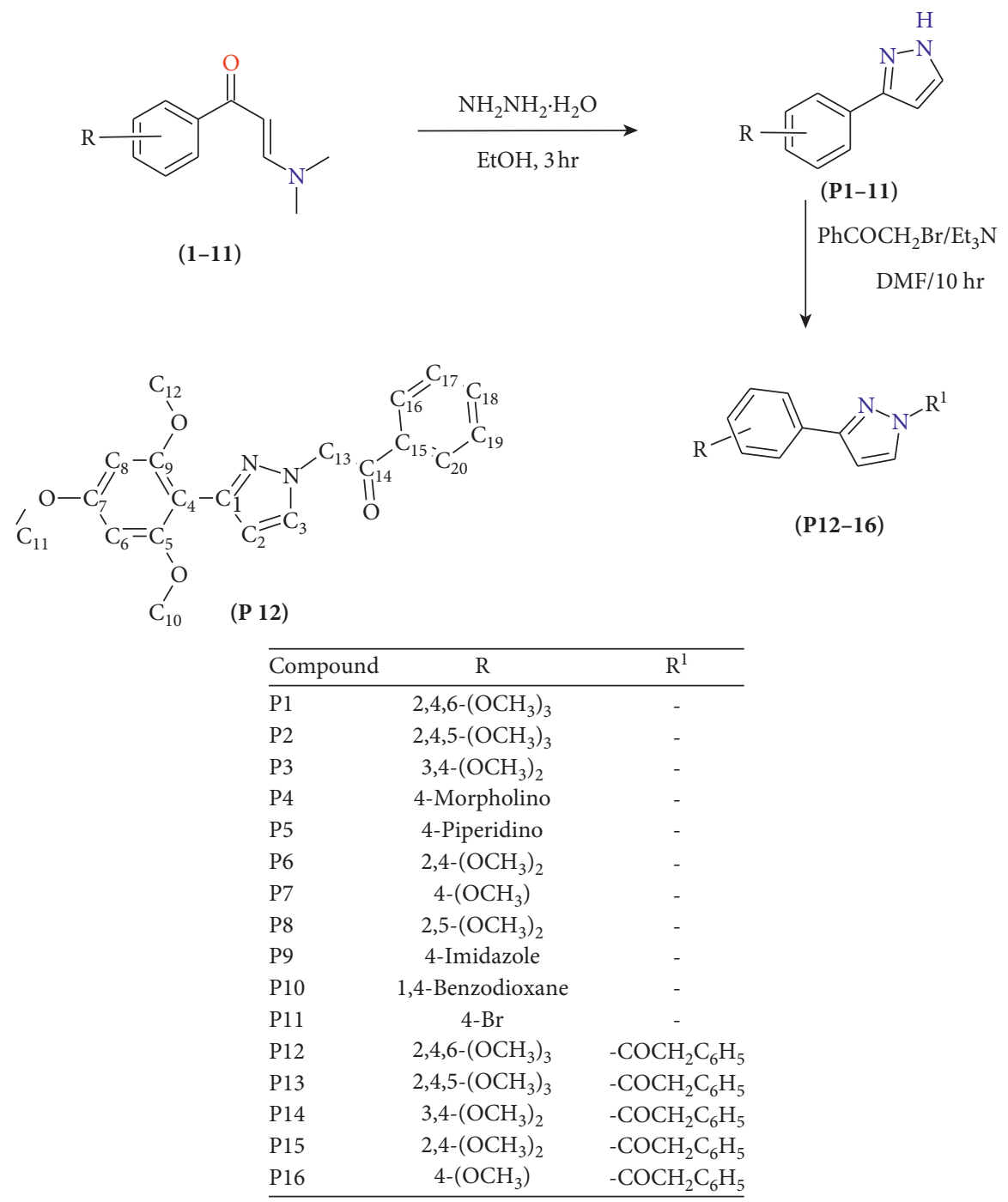

Scheme 1: Synthetic route of compounds (P1-P16).

McFarland turbidity standards. The activity was performed on Mueller-Hinton agar plates. The bacteria were spread over agar plates, and the $5 \mathrm{~mm}$ wells were created aseptically in the agar plate. The $20 \mu \mathrm{L}$ of each tested compound $(250 \mu \mathrm{g} / \mathrm{mL})$ was added to wells, and plates were incubated overnight at $37^{\circ} \mathrm{C}$. The zone of inhibition was measured, and comparative activity of synthesized compounds was determined.

2.7. Antibacterial Activity at Different Time Points. The synthesized compounds were solubilized in DMSO with initial concentration of $5 \mu \mathrm{g} / \mu \mathrm{L}$. This initial stock was used to adjust different concentrations of tested compounds. The concentration was adjusted in Mueller-Hinton broth as 500, $300,200,100,50$, and $0 \mu \mathrm{g} / \mathrm{mL}$.

2.8. Antimicrobial Activity of Compounds through Optical Density. Antibacterial activity of tested compounds against ATCC 6538, NCTC 10400, NCTC 10418, and ATCC 27853 was determined by analysis of bacterial growth at different time points under the influence of different concentrations of tested compounds. The bacterial growth was determined in terms of optical density at $600 \mathrm{~nm}$. The $\mathrm{OD}_{600}$ was measured through a microplate reader (BioTek, USA). The $10^{-3}$ diluted active bacterial culture was prepared from overnight growth of bacteria in Mueller-Hinton agar, and $5 \mathrm{~mL}$ of this diluted bacterial culture was added to a 96-well plate containing different concentrations of tested compounds. This plate was incubated at $37^{\circ} \mathrm{C}$, and $\mathrm{OD}_{600}$ was measured at different time points ranging from 0 to 6 hours. The result was prepared by plotting graph between time vs. $\mathrm{OD}_{600}$. The resultant curve was used to analyze effects of tested compounds on the growth of bacteria. All $\mathrm{OD}_{600}$ readings were calculated in terms of $\mathrm{OD}_{600}(\mathrm{t})-$ $\mathrm{OD}_{600}(0 \mathrm{~h})$, where " $\mathrm{t}$ " represent for time points (h). Gentamicin (Gibco) was used as positive control for bacterial growth inhibition; in contrast, the bacterial growth in the absence of synthesized compounds was used as negative control. 
TABLE 1: Zone of inhibition of synthesized compounds $(250 \mu \mathrm{g} / \mathrm{mL})$ and standard drug gentamicin $(250 \mu \mathrm{g} / \mathrm{mL})$ against Gram-positive and Gram-negative bacteria after $24 \mathrm{hrs}$.

\begin{tabular}{lcc}
\hline Compound & E. coli & S. aureus \\
\hline P1 & 0 & 1.48 \\
P6 & 0 & 1.21 \\
P11 & 1.90 & 2.63 \\
Gentamicin & 2.50 & 2.90 \\
\hline
\end{tabular}

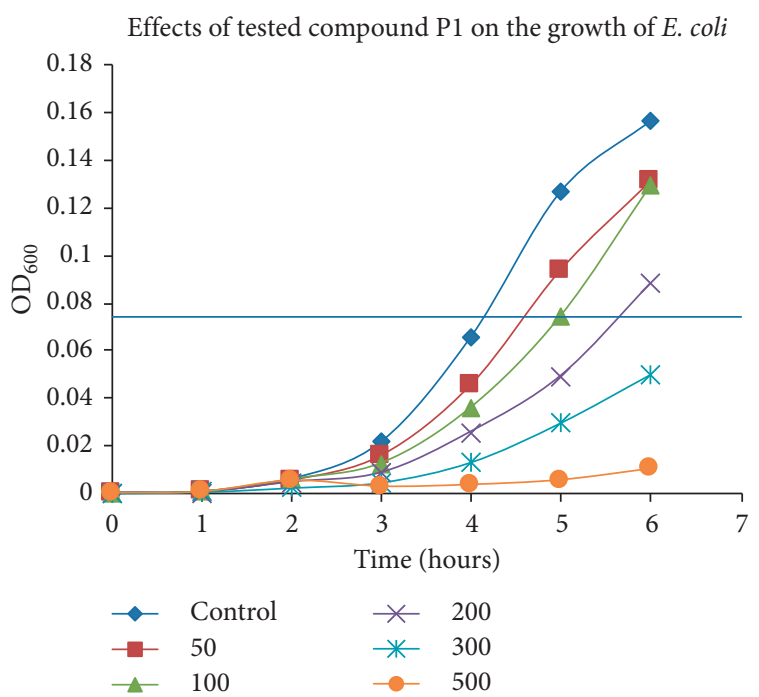

(a)

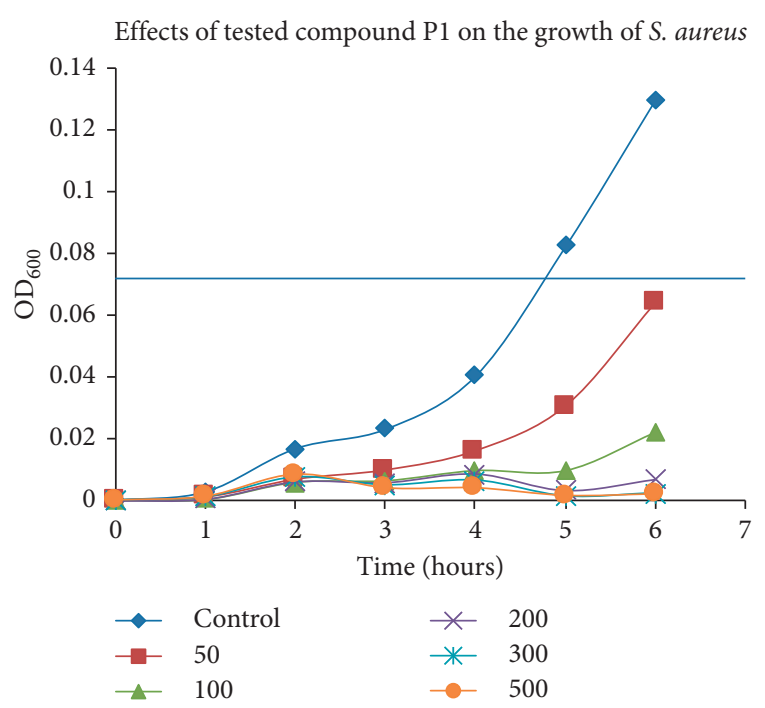

(c)

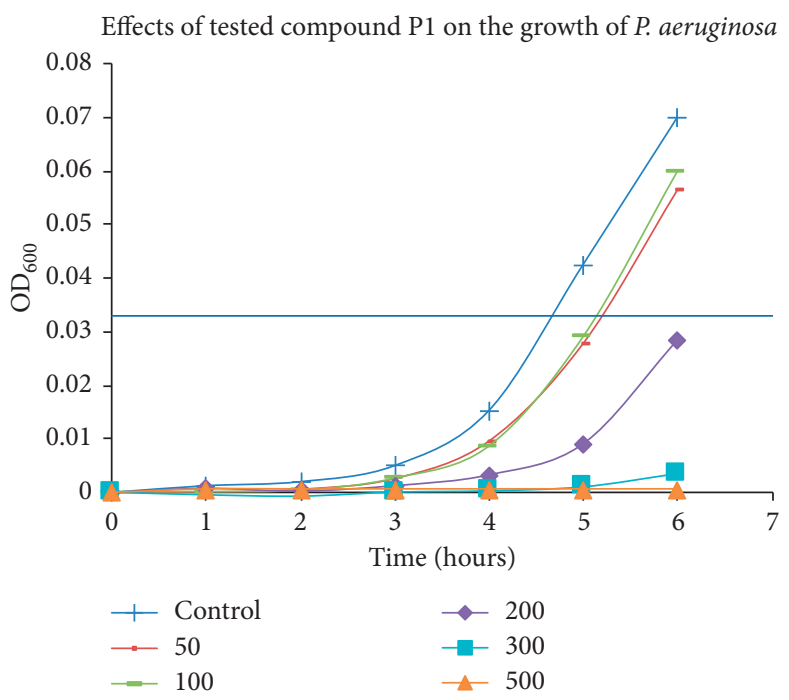

(b)

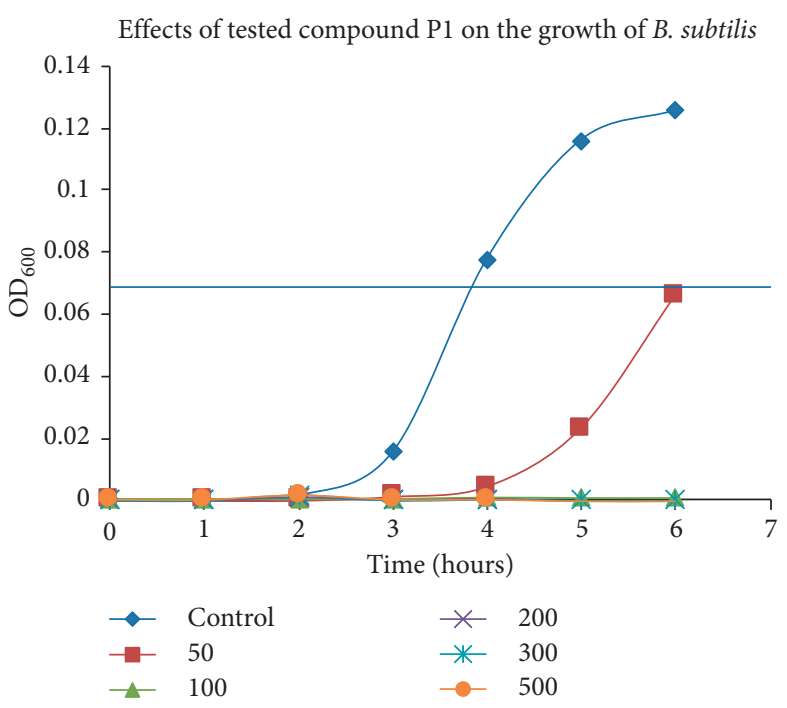

(d)

Figure 1: Effects of different concentrations of compound $\mathbf{P 1}$ against different time points. All compound concentrations are in $\mu \mathrm{g} / \mathrm{mL}$.

\section{Results and Discussion}

Enaminone derivatives (1-11) were used as a starting material for the synthesis of various pyrazole derivatives (P1-P16). Various enaminones were obtained by the reaction of substituted acetophenones with DMF-DMA in a solvent-free environment [35, 36]. Pyrazole derivatives (P1-P11) were obtained, when substituted enaminones were reacted with hydrazine hydrate $99 \%$ in presence of absolute alcohol. $N$-substituted pyrazole derivatives (P12P16) were obtained by reacting pyrazole derivatives with phenacyl bromide in dimethylformamide (DMF) in presence of triethylamine. The synthesis of these compounds was performed through an efficient synthetic route (Scheme1). The structures of all the synthesized compounds were confirmed by proton and carbon NMR spectroscopy. ${ }^{1} \mathrm{H}$ 


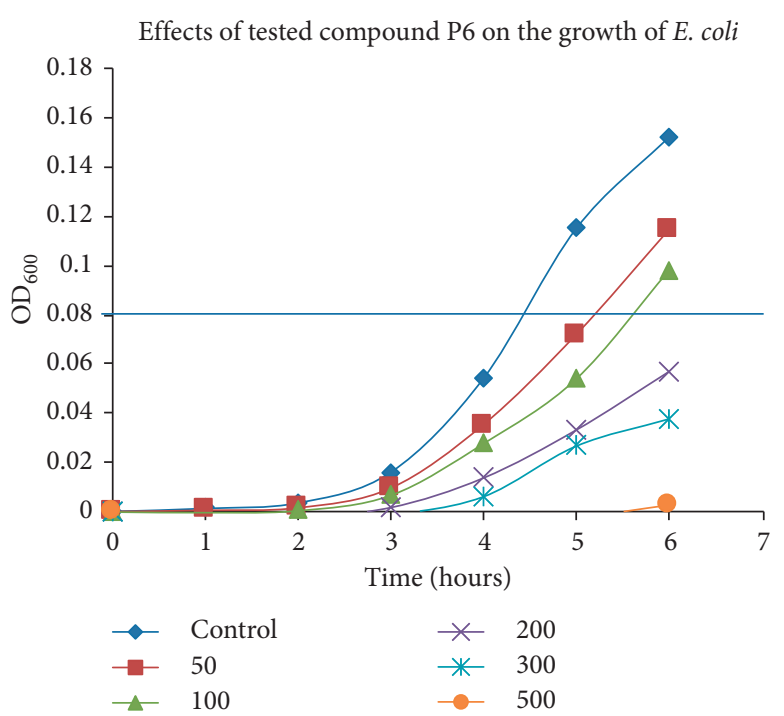

(a)

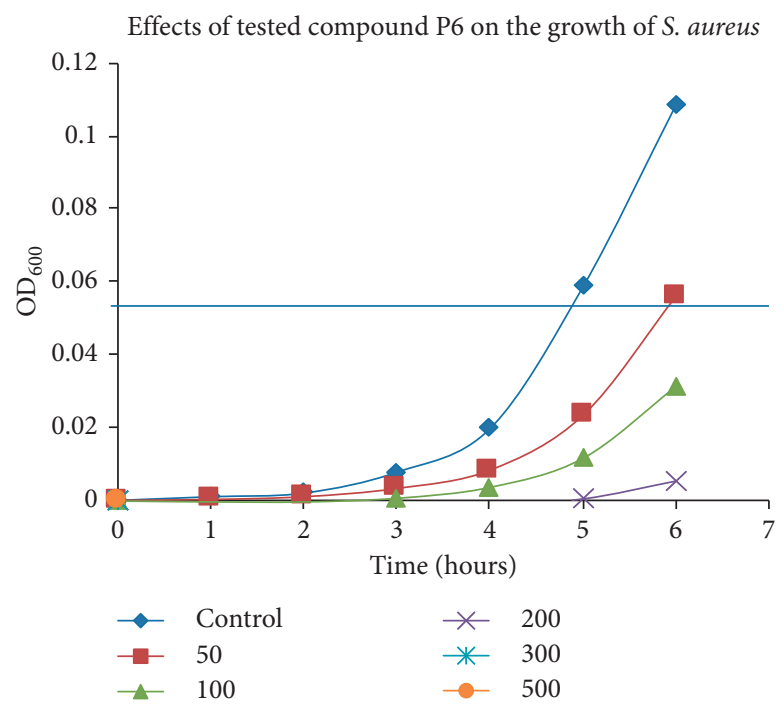

(c)

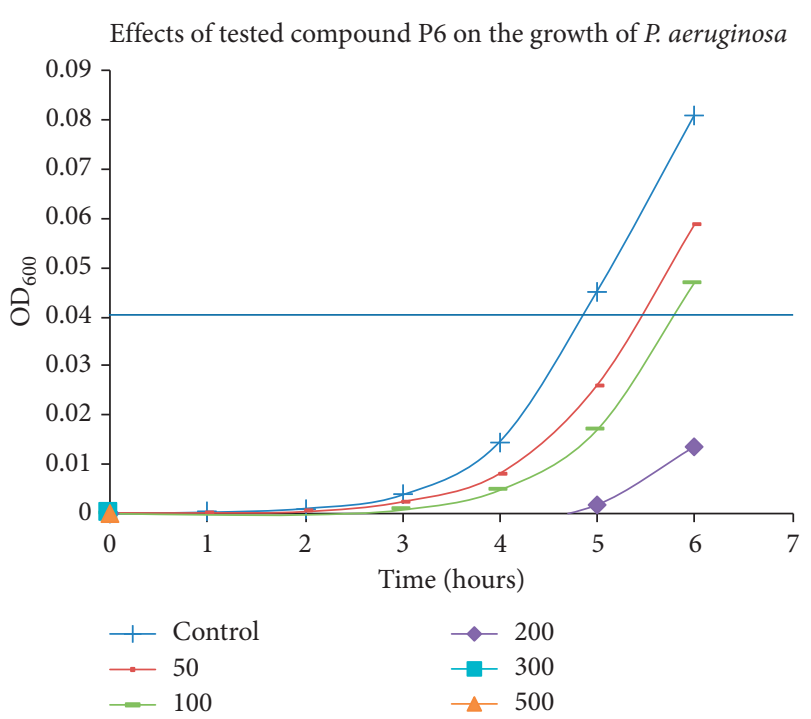

(b)

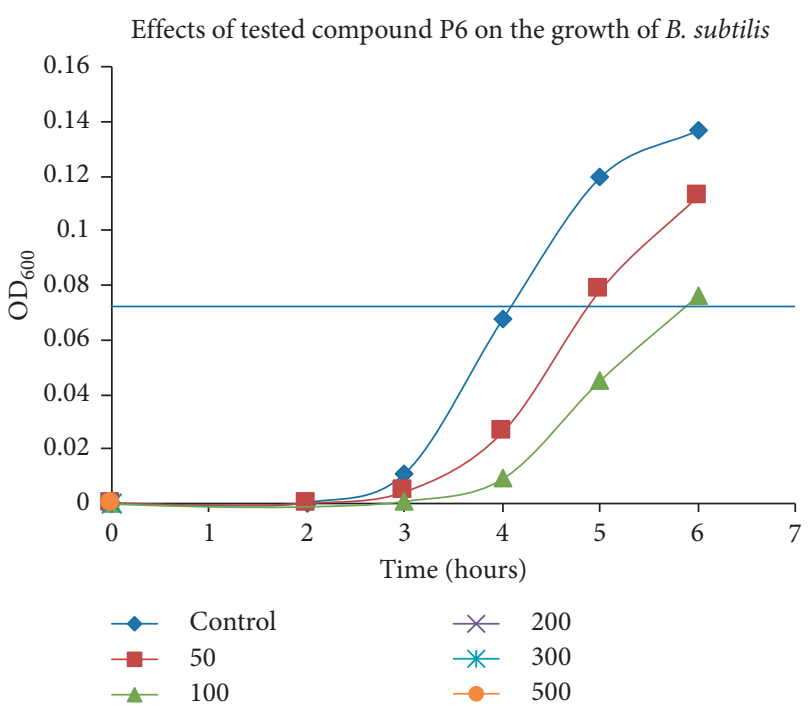

(d)

Figure 2: Effects of different concentrations of compound $\mathbf{P 6}$ against different time points. All compound concentrations are in $\mu \mathrm{g} / \mathrm{mL}$.

NMR spectrum of (1) displayed two doublets at $\delta \mathrm{H}$ $5.80-5.82$ and $7.63-7.65 \mathrm{ppm}(\mathrm{d}, J=14 \mathrm{~Hz})$ due to the ethylenic protons. The value of coupling constant $(J=14 \mathrm{~Hz})$ for the ethylenic protons indicates that the enaminones existed in the E-configuration which was also confirmed by single-crystal X-ray crystallography [37]. All the pyrazoles presented the $\mathrm{D}_{2} \mathrm{O}$ exchangeable broad singlet at $\delta \mathrm{H}$ $11.8-13.00 \mathrm{ppm}$ corresponding to the NH proton. The aromatic protons appeared at $\delta \mathrm{H} 6.0-8.32 \mathrm{ppm}$. The two pyrazole protons appeared as doublets with $J=7 \mathrm{~Hz}$ at $\delta \mathrm{H}$ $6.62-7.47 \mathrm{ppm}$ and $\delta \mathrm{H}$ 6.66-7.79 ppm. In case of $N$ substituted pyrazoles, $\left(\mathrm{OCH}_{3}\right)$ protons were observed at $\delta \mathrm{H}$ $3.69-3.86 \mathrm{ppm}$. The two pyrazole protons appeared as doublets with $J=7 \mathrm{~Hz}$ at $\delta \mathrm{H}$ 6.19-6.79 ppm and $\delta \mathrm{H}$ $6.27-7.40 \mathrm{ppm}$. The aromatic protons appeared at $\delta \mathrm{H}$ $7.32-8.00 \mathrm{ppm}$. The $\mathrm{CH}_{2}$ protons were observed at $\delta \mathrm{H}$ 5.80-5.90. The presence of all carbon atoms for all the compounds was confirmed by ${ }^{13} \mathrm{C}$ NMR spectra. Molecular weights of compounds were confirmed by GC/MS spectroscopy. The detailed spectral results of ${ }^{1} \mathrm{H}$ NMR spectra, ${ }^{13} \mathrm{C}$ NMR spectra, and mass spectra are given in the experimental part. The spectral and analytical data confirmed the composition of the synthesized compounds (P1-P16).

3.1. Disc Diffusion Assay. The antimicrobial activity of all the synthesized compounds was determined by disc diffusion assay, and their data are presented in Table 1. Only three compounds (P1, P6, and P11) showed good antimicrobial activity.

3.2. Bacterial Growth Inhibition at Different Time Points. The compounds (P1, P6, and P11), selected in initial screening steps, were further evaluated for their 


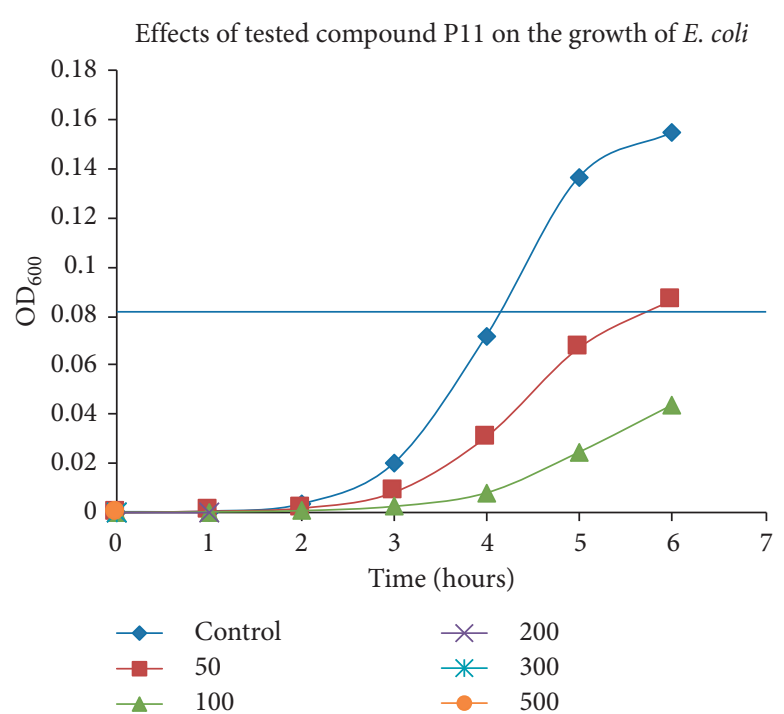

(a)

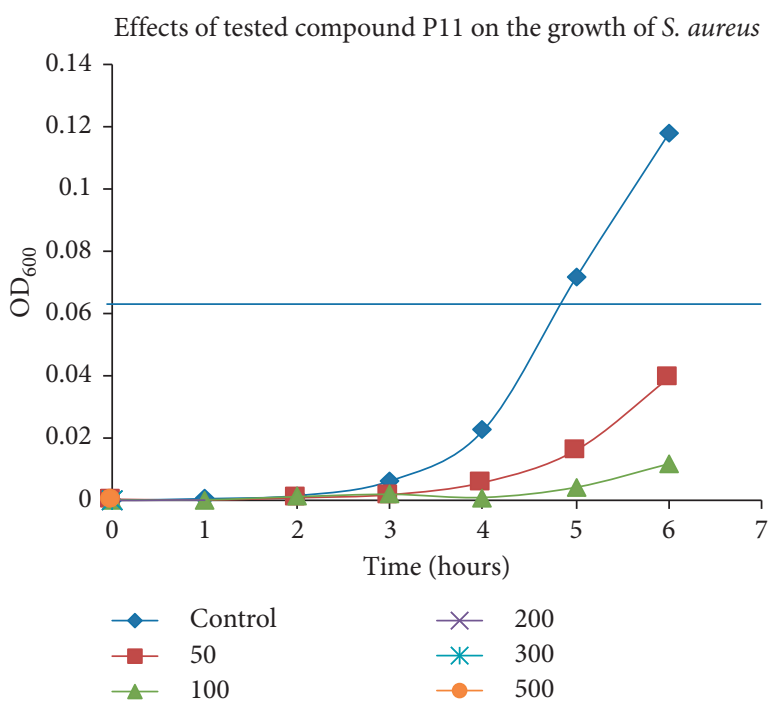

(c)

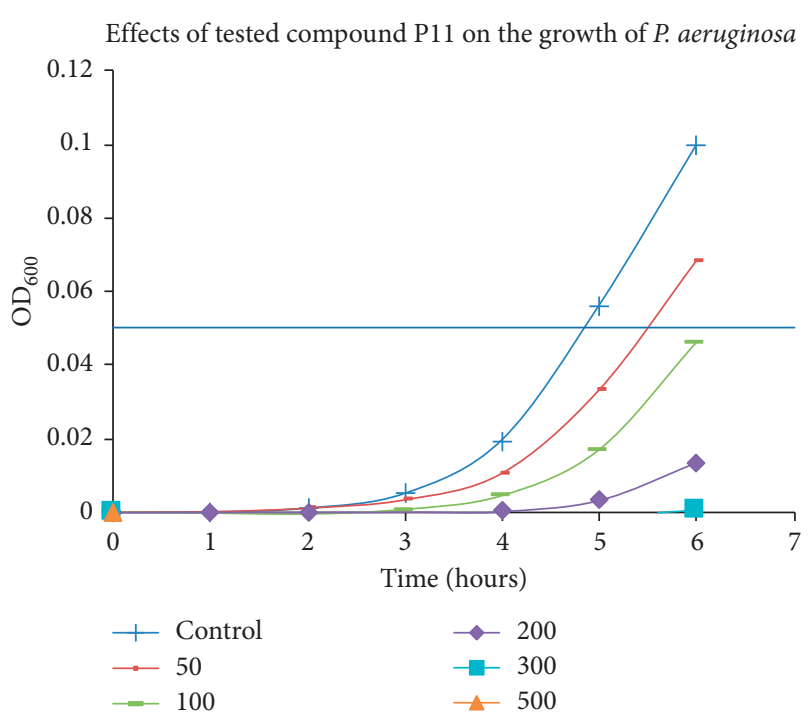

(b)

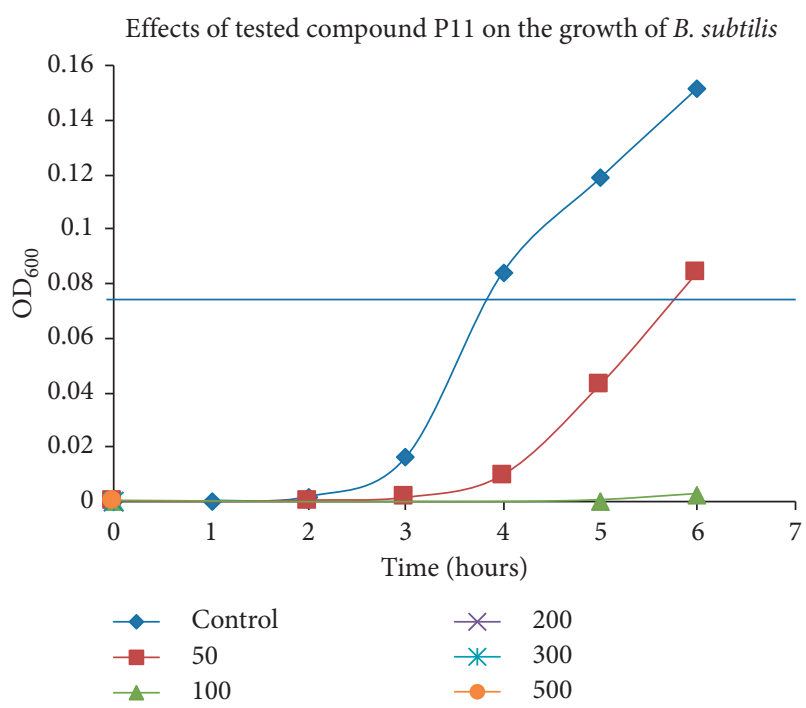

(d)

Figure 3: Effects of different concentrations of compound P11 against different time points. All compound concentrations are in $\mu \mathrm{g} / \mathrm{mL}$.

antimicrobial activity at different time points. The data of these compounds against tested bacteria are presented in Figures 1-4. The concentration of the compound inhibiting $50 \%$ growth in comparison with control after 6 hours was also calculated, and data are presented in Table 2. All the synthesized compounds were screened, and only three compounds (P1, P6, and P11) showed antimicrobial activity during the screening step through disc diffusion assay. Among these three active compounds, only one compound (P11) showed antimicrobial activity against both Grampositive and Gram-negative bacteria after 24 hours. All the three compounds were showing good antimicrobial activity against Gram-positive bacteria. Compound P11 presented maximum activity among all active compounds against both Gram-positive and Gram-negative bacteria. The compounds were tested for antimicrobial activity at different time points against Gram-positive and Gram-negative bacteria; it was found that all of these compounds were showing good activity against Gram-positive and Gram-negative bacteria for 6 hours, but the activity was higher against Gram-positive bacteria. In contrast, at 24 hours, the activity was found only against Gram-positive bacteria except one compound (P11), showing activity against both bacteria. The reason for this discrepancy may be due to many reasons. Limited efficacy of compounds may be present for certain time only and can give result at 6 hours but not at 24 hours. In addition, degradation of compounds in DMSO solvent was checked for 24 hours. It was found that all the compounds were stable. Moreover, mechanism for resistance in Gram-negative bacteria is different than Gram-positive bacteria that may also cause these results. However, the water solubility of these compounds was very poor, and therefore, we used DMSO as cosolvent and performed this study as per the given protocol [38]. Gentamicin showed very good 


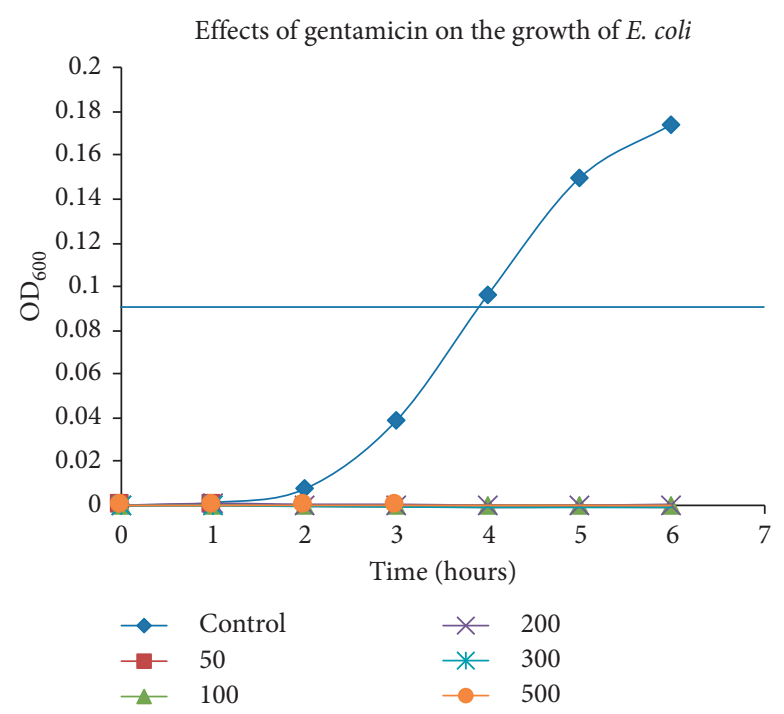

(a)

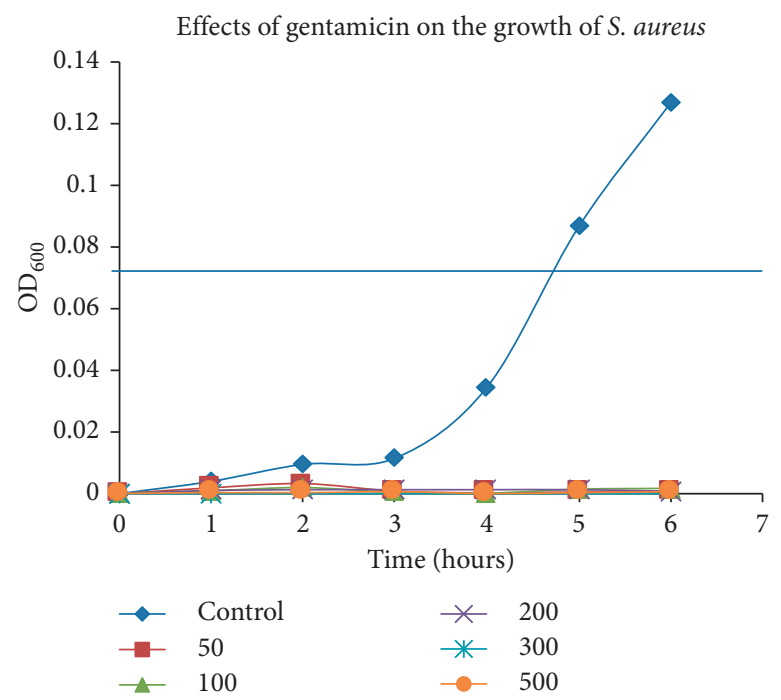

(c)

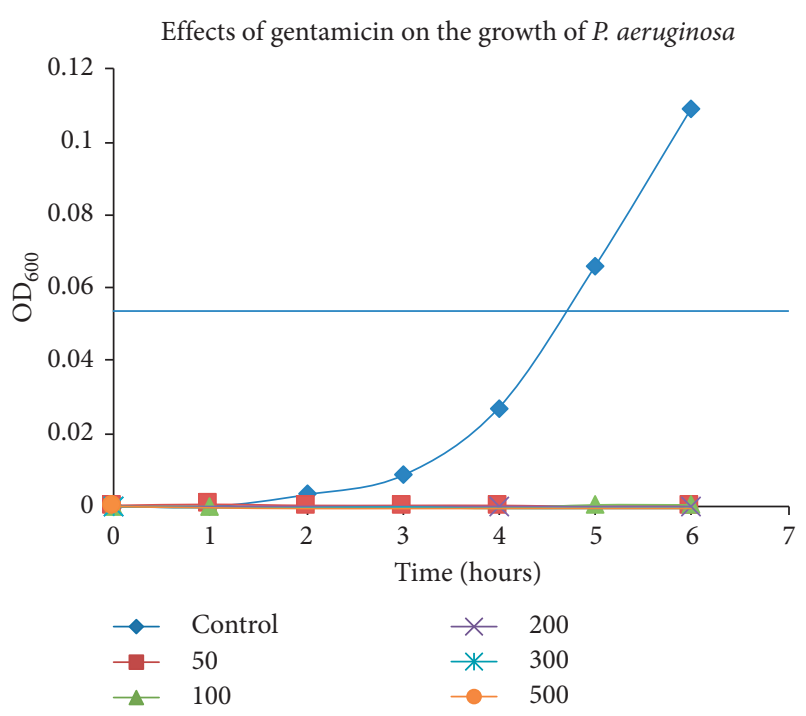

(b)

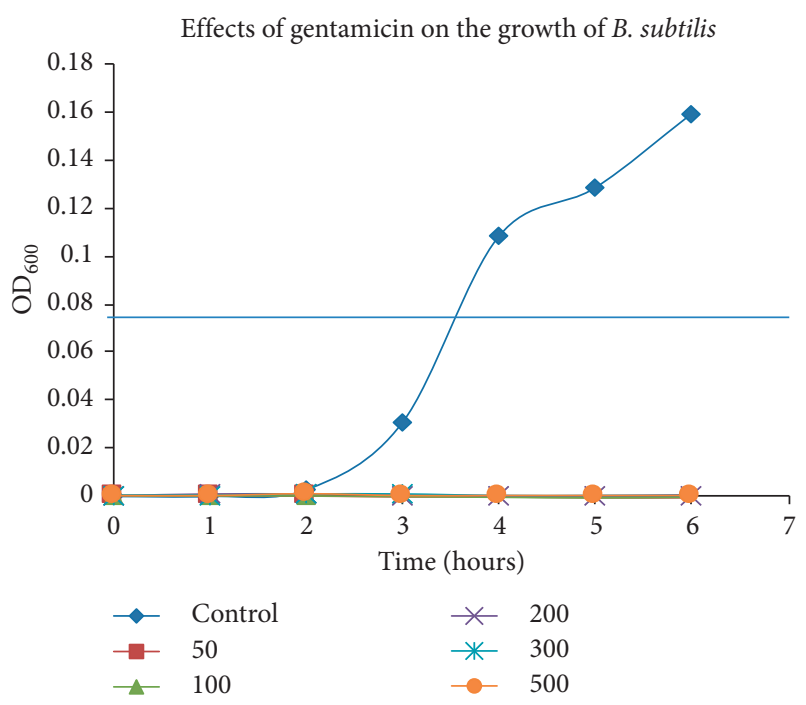

(d)

FIGURE 4: Effects of different concentrations of gentamicin against different time points. All compound concentrations are in $\mu \mathrm{g} / \mathrm{mL}$.

TABLE 2: Concentration of compounds required to reduce bacterial growth by $50 \%$ in comparison with control gentamicin.

\begin{tabular}{lcccc}
\hline Compound & & & S. aureus & \\
\hline P1 & E. coli & P. aeruginosa & $<50$ & B. subtilis \\
P6 & $<100$ & $<100$ & $<50$ & $<50$ \\
P11 & $<100$ & $<100$ & $<50$ & $<100$ \\
Gentamicin & $<50$ & $<50$ & $<50$ & $<50$ \\
\hline
\end{tabular}

${ }^{*}$ All concentrations are in $\mu \mathrm{g} / \mathrm{mL}$.

antimicrobial activity against tested compounds, and it was not allowing any growth of microbes even at least concentration.

\section{Conclusion}

In conclusion, a series of pyrazoles and $N$-substituted pyrazoles were synthesized in good yield from enaminones by an efficient synthetic route. All the synthetic compounds were characterized by spectral data and elemental analysis. Antimicrobial activity of the compounds was determined by the disc diffusion assay. In the initial screening, only compounds (P1, P6, and P11) showed significant antimicrobial activity. These three compounds were further evaluated for their antimicrobial activity at different time points. Compound number P11 presented maximum activity 
among all active compounds against both Gram-positive and Gram-negative bacteria. Active compounds were screened for antimicrobial activity at different time points against Gram-positive and Gram-negative bacteria, and it was found that all of these compounds were showing good activity against Gram-positive and Gram-negative bacteria for 6 hours, but the activity was higher against Grampositive bacteria. In contrast, at 24 hours, the activity was found only against Gram-positive bacteria except one compound (P11) showing activity against both types of bacteria.

\section{Data Availability}

Samples of the compounds (P1-P16) in pure form are available from the authors.

\section{Conflicts of Interest}

The authors declare no conflicts of interest.

\section{Acknowledgments}

The authors would like to extend their sincere appreciation to the Deanship of Scientific Research at King Saud University for funding this research group (no. RG 1435-006).

\section{Supplementary Materials}

The spectral data of compounds (P1-P16) used to support the findings of this study are included within the supplementary information file. (Supplementary Materials)

\section{References}

[1] I. O. Edafiogho, S. B. Kombian, K. V. V. Ananthalakshmi et al., "Enaminones: exploring additional therapeutic activities," Journal of Pharmaceutical Sciences, vol. 96, no. 10, pp. 2509-2531, 2007.

[2] H. M. Gaber, M. C. Bagley, Z. A. Muhammada, and S. M. Gomha, "Recent developments in chemical reactivity of $\mathrm{N}, \mathrm{N}$-dimethylenamino ketones as synthons for various heterocycles. RSC Adv. 7, 14562-14610," Chemical Society Reviews, vol. 6, pp. 277-294, 2017.

[3] I. Chaaban, J. V. Greenhill, and P. Akhtar, "Enaminones in the mannich reaction. Part 2. Further investigations of internal mannich reactions," Journal of the Chemical Society, Perkin Transactions 1, vol. 6, pp. 1593-1596, 1979.

[4] J. P. Michael, C. B. De Koning, D. Gravestock et al., "Enaminones: versatile intermediates for natural product synthesis," Pure and Applied Chemistry, vol. 71, no. 6, pp. 979-988, 1999.

[5] A. C. Spivey, R. Srikaran, C. M. Diaper, and D. J. Turner, "Traceless solid phase synthesis of 2-substituted pyrimidines using an "off-the-shelf" chlorogermane-functionalised resin," Organic and Biomolecular Chemistry, vol. 1, no. 10, pp. 1638-1640, 2003.

[6] F. R. Souza, V. T. Souza, V. Ratzlaff et al., "Hypothermic and antipyretic effects of 3-methyl- and 3-phenyl-5-hydroxy-5trichloromethyl-4,5-dihydro-1H-pyrazole-1-carboxyamides in mice," European Journal of Pharmacology, vol. 451, no. 2, pp. 141-147, 2002.
[7] Y. F. Wang, T. Izawa, S. Kobayashi, and M. Ohno, "Stereocontrolled synthesis of (+)-negamycin from an acyclic homoallylamine by 1,3-asymmetric induction," Journal of the American Chemical Society, vol. 104, no. 23, pp. 6465-6466, 1982.

[8] J. E. Foster, J. M. Nicholson, R. Butcher et al., "Synthesis, characterization and anticonvulsant activity of enaminones. Part 6: synthesis of substituted vinylic benzamides as potential anticonvulsants," Bioorganic \& Medicinal Chemistry, vol. 7, no. 11, pp. 2415-2425, 1999.

[9] J. P. Michael, C. B. De Koning, G. D. Hosken, and T. V. Stanbury, "Reformatsky reactions with N-arylpyrrolidine-2-thiones: synthesis of tricyclic analogues of quinolone antibacterial agents," Tetrahedron, vol. 57, no. 47, pp. 9635-9648, 2001.

[10] D. L. Boger, T. Ishizaki, R. J. Wysocki, S. A. Munk, P. A. Kitos, and O. Suntornwat, "Total synthesis and evaluation of (.+-.)-N-(tert-butoxycarbonyl)-CBI, (.+-.)-CBI-CDPI1, and (.+-.)-CBI-CDPI2: CC-1065 functional agents incorporating the equivalent $1,2,9,9$ a-tetrahydrocyclopropa[1,2-c]benz[1,2e]indol-4-one (CBI) left-hand subunit," Journal of the American Chemical Society, vol. 111, no. 16, pp. 6461-6463, 1989.

[11] H. M. C. Ferraz and E. R. S. Gonçalo, "Preparações e aplicações sintéticas recentes de enaminonas," Química Nova, vol. 30, no. 4, pp. 957-964, 2007.

[12] A.-Z. A. Elassar and A. A. El-Khair, "Recent developments in the chemistry of enaminones," Tetrahedron, vol. 59, no. 43, pp. 8463-8480, 2003.

[13] M. V. Patel, R. Bell, S. Majest, R. Henry, and T. Kolasa, "Synthesis of 4,5-diaryl-1H-pyrazole-3-ol derivatives as potential COX-2 inhibitors," The Journal of Organic Chemistry, vol. 69, no. 21, pp. 7058-7065, 2004.

[14] P. K. Sharma, S. Kumar, P. Kumar et al., "Synthesis and biological evaluation of some pyrazolylpyrazolines as antiinflammatory-antimicrobial agents," European Journal of Medicinal Chemistry, vol. 45, no. 6, pp. 2650-2655, 2010.

[15] A. F. Sharif, M. A. Rostom, M. A. Shalaby, and El-Demellawy, "Polysubstituted pyrazoles, part 5. Synthesis of new 1-(4chlorophenyl)-4-hydroxy-1H-pyrazole-3-carboxylic acid hydrazide analogs and some derived ring systems. A novel class of potential antitumor and anti-HCV agents," European Journal of Medicinal Chemistry, vol. 38, pp. 959-974, 2003.

[16] D. Nikhila, K. P. Amnekar, and Bhusari, "Synthesis, anticonvulsant activity and 3D-QSAR study of some prop-2eneamido and 1-acetyl-pyrazolin derivatives of aminobenzothiazole," European Journal of Medicinal Chemistry, vol. 45, pp. 149-159, 2010.

[17] Z. Özdemir, H. B. Kandilci, B. Gümüşel, Ü. Çalış, and A. A. Bilgin, "Synthesis and studies on antidepressant and anticonvulsant activities of some 3-(2-furyl)-pyrazoline derivatives," European Journal of Medicinal Chemistry, vol. 42, no. 3, pp. 373-379, 2007.

[18] M. J. Genin, C. Biles, B. J. Keiser et al., "Novel 1,5-diphenylpyrazole nonnucleoside HIV-1 reverse transcriptase inhibitors with enhanced activity versus the delavirdineresistant P236L mutant: lead identification and SAR of 3- and 4-substituted derivatives," Journal of Medicinal Chemistry, vol. 43, no. 5, pp. 1034-1040, 2000.

[19] R. S. Joshi, P. G. Mandhane, S. D. Diwakar, S. K. Dabhade, and C. H. Gill, "Synthesis, analgesic and anti-inflammatory activities of some novel pyrazolines derivatives," Bioorganic \& Medicinal Chemistry Letters, vol. 20, no. 12, pp. 3721-3725, 2010. 
[20] C. W. Noell and C. C. Cheng, "Pyrazoles. 4. Analogs of 3-(3,3dimethyl-1-triazeno)pyrazole-4-carboxamide," Journal of Medicinal Chemistry, vol. 14, no. 12, pp. 1245-1246, 1971.

[21] M. Abdel-Aziz, G. E.-D. A. Abuo-Rahma, and A. A. Hassan, "Synthesis of novel pyrazole derivatives and evaluation of their antidepressant and anticonvulsant activities," European Journal of Medicinal Chemistry, vol. 44, no. 9, pp. 3480-3487, 2009.

[22] S. Shelke, G. Mhaske, S. Gadakh, and C. Gill, "Green synthesis and biological evaluation of some novel azoles as antimicrobial agents," Bioorganic \& Medicinal Chemistry Letters, vol. 20, no. 24, pp. 7200-7204, 2010.

[23] M. A. Bhat, A. A. Khan, M. A. Al-Omar, and A. A. Khan, "Synthesis and anti-candidal activity of some new pyrazoline derivatives," Biomedical Research, vol. 28, pp. 3082-3087, 2017.

[24] M. Bhat, A. Al-Dhfyan, and M. Al-Omar, "Targeting cancer stem cells with novel 4-(4-substituted phenyl)-5-(3,4,5-trimethoxy/3,4-dimethoxy)-benzoyl-3,4-dihydropyrimidine2(1H)-one/thiones," Molecules, vol. 21, no. 12, p. 1746, 2016.

[25] A. A. Hassan, "Heterocyclic synthesis via enaminones: synthesis and molecular docking studies of some novel heterocyclic compounds containing sulfonamide moiety," International Journal of Organic Chemistry, vol. 4, pp. 68-81, 2014.

[26] H. V. Chavan, L. K. Adsul, A. S. Kotmale, V. D. Dhakane, V. N. Thakare, and B. P. Bandgar, "Design, synthesis, characterization andin vitroandin vivoanti-inflammatory evaluation of novel pyrazole-based chalcones," Journal of Enzyme Inhibition and Medicinal Chemistry, vol. 30, no. 1, pp. 22-31, 2015.

[27] R. Cantegril, D. Croisat, P. Desbordes et al., "Fungicidal arylpyrazoles and their preparation, compositions, and use," Patent Cooperation Treaty WO 9322287 A1 19931111, 1993.

[28] H. Zhu, C. Wang, Z. Wang, and L. Shi, "For preparation of antitumor pyrazoles compounds containing amide bonds," Faming Zhuanli Shenqing CN 106986860 A 20170728, 2017.

[29] A. Kling, K. Jantos, H. Mack et al., "Preparation of heterocyclic carboxamide compounds and their use as therapeutic calpain inhibitors," United States Patent US 20110152265 A1 20110623, 2011.

[30] C. Lopez, R. M. Claramunt, S. Trofimenko, and J. Elguero, "A 13C NMR spectroscopy study of the structure of N-H pyrazoles and indazoles," Canadian Journal of Chemistry, vol. 71, no. 5, pp. 678-684, 1993.

[31] J. Zhang, J.-F. Peng, T. Wang, Y. Kang, S. Jing, and Z.-T. Zhang, "Synthesis and biological evaluation of arylpyrazoles as fungicides against phytopathogenic fungi," Molecular Diversity, vol. 21, no. 2, pp. 317-323, 2017.

[32] M. A. Halcrow, H. R. Powell, and M. Duer, "Tautomerism in 3 $\{5\}$-(dimethoxyphenyl) pyrazoles," Acta Crystallographica Section B, vol. B52, pp. 746-752, 1996.

[33] Z.-B. Huang, L.-L. Li, Y.-W. Zhao, H.-Y. Wang, and D.-Q. Shi, "An efficient synthesis of isoxazoles and pyrazoles under ultrasound irradiation," Journal of Heterocyclic Chemistry, vol. 51, no. S1, pp. E309-E313, 2014.

[34] M. Balouiri, M. Sadiki, and S. K. Ibnsouda, "Methods for in vitro evaluating antimicrobial activity: a review," Journal of Pharmaceutical Analysis, vol. 6, no. 2, pp. 71-79, 2016.

[35] M. Bhat, M. Al-Omar, H. Ghabbour, and A. Naglah, "A onepot biginelli synthesis and characterization of novel dihydropyrimidinone derivatives containing piperazine/morpholine moiety," Molecules, vol. 23, no. 7, p. 1559, 2018.
[36] M. A. Bhat, M. A. Al-Omar, H. Ghabbour, and A. M. Naglah, "Synthesis and in vivo anti-ulcer evaluation of some novel piperidine linked dihydropyrimidinone derivatives," Journal of Enzyme Inhibition and Medicinal Chemistry, vol. 33, pp. 978-988, 2018.

[37] M. A. Bhat, A. F. Ahmed, Z.-H. Wen, M. A. Al-Omar, and H. A. Abdel-Aziz, "Synthesis, anti-inflammatory and neuroprotective activity of pyrazole and pyrazolo[3,4-d]pyridazine bearing 3,4,5-trimethoxyphenyl," Medicinal Chemistry Research, vol. 26, no. 7, pp. 1557-1566, 2017.

[38] M. Khan, S. T. Khan, M. Khan et al., "Antibacterial properties of silver nanoparticles synthesized using Pulicaria glutinosa plant extract as a green bioreductant," International Journal of Nanomedicine, vol. 9, pp. 3551-3565, 2014. 

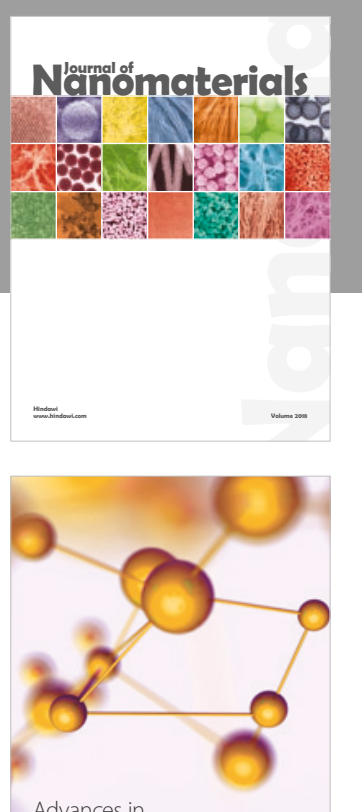

Physical Chemistry
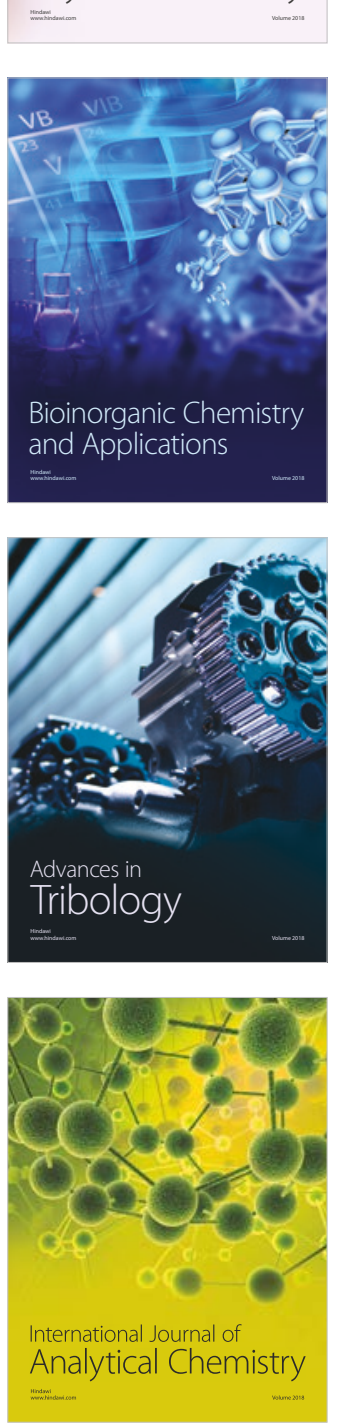

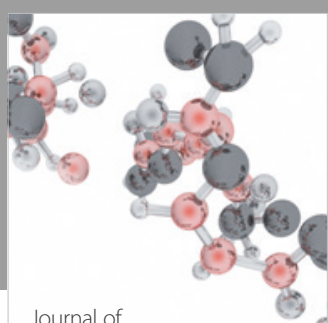

Analytical Methods

in Chemistry

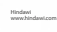

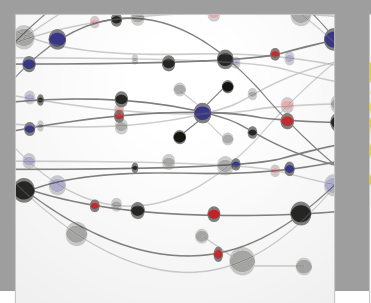

The Scientific World Journal

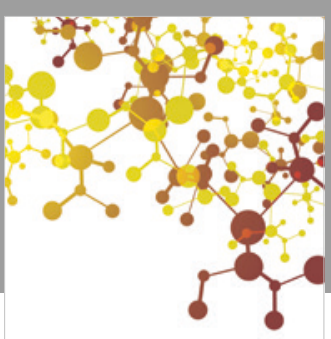

Journal of

Applied Chemistry
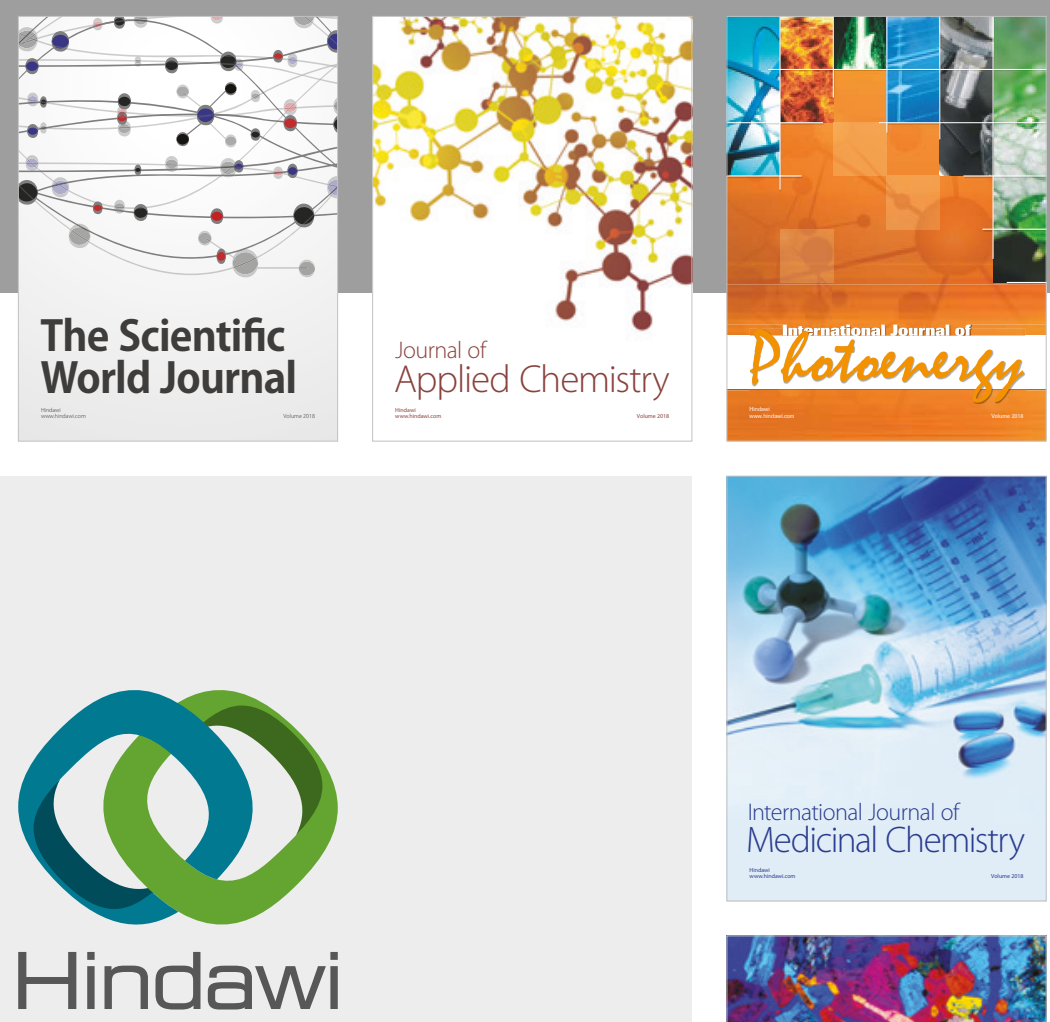

Submit your manuscripts at

www.hindawi.com
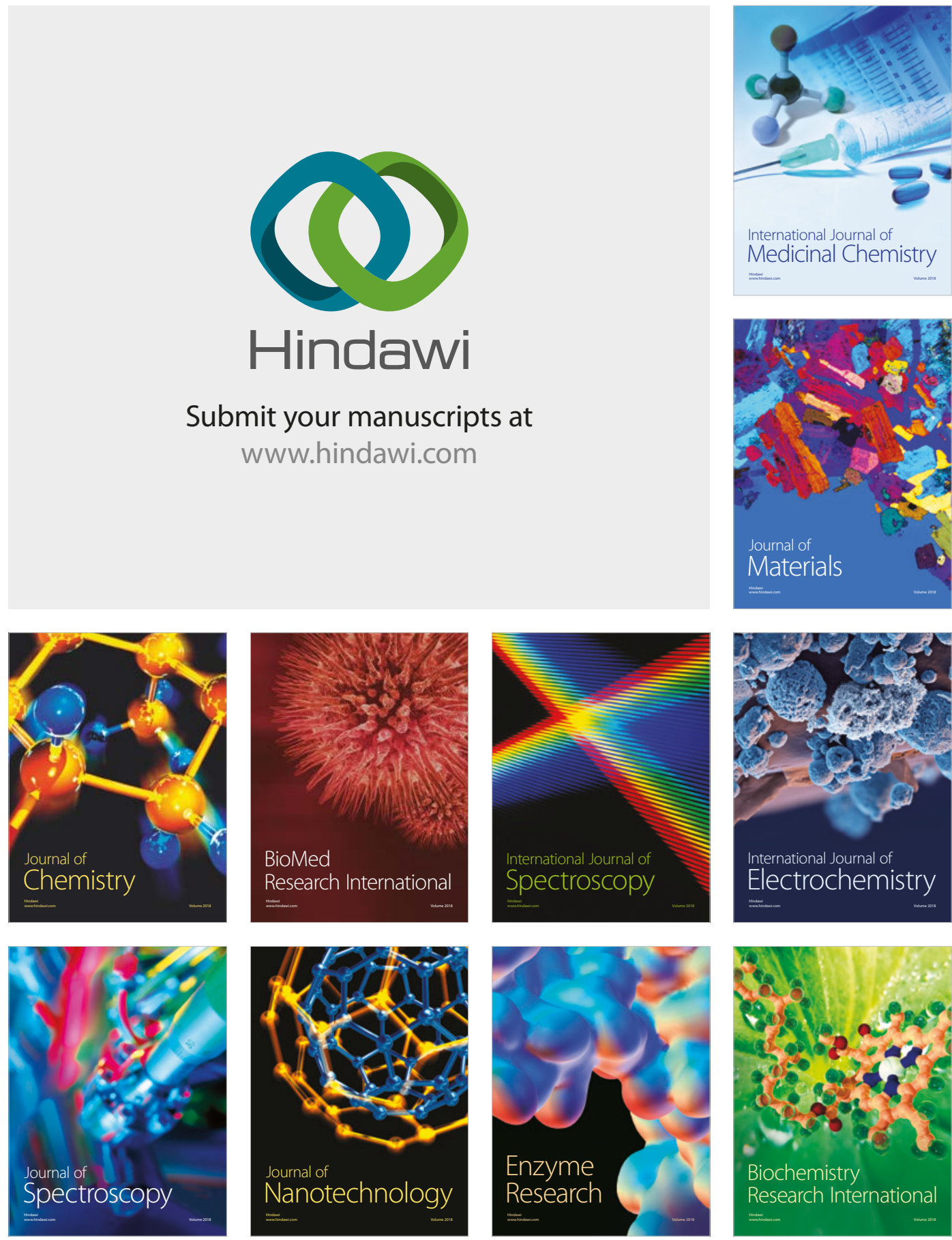
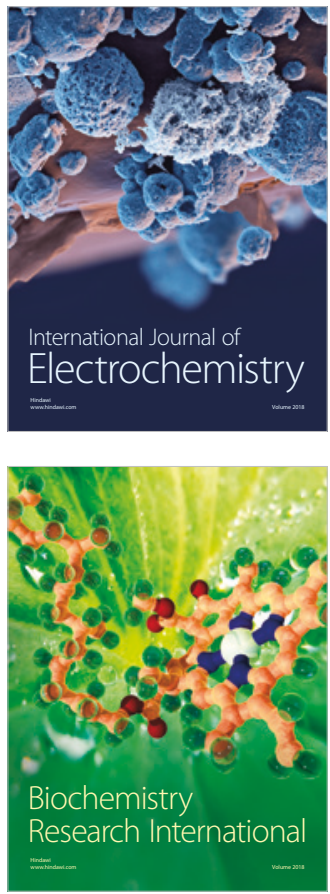